\title{
ACTIVIDAD ALEGATORIA, PRINCIPIO DISPOSITIVO Y PRUEBA DE OFICIO (CORTE DE APELACIONES DE CONCEPCIÓN)
}

\author{
Comentario de Iván Hunter Ampuero
}

Concepción, siete de julio de dos mil nueve.

VISTOS:

A fojas 47 el abogado don Fernando Saenger Gianoni, en representación de Ingecol S.A., dedujo demanda en contra de Serviu Región del Bío-Bío, esgrimiendo que las partes consintieron, la primera como contratista y la segunda como mandante, en la ejecución de la obra denominada "Mejoramiento Vial, Eje Los Carrera, Segunda Etapa, Concepción, entre Janequeo y General Novoa, por el precio de \$1.709.283.314, y, sosteniendo que el servicio público demandado habría incurrido en diversas formas de incumplimientos contractuales, pide que se le condene a pagar a la actora las cifras de $\$ 166.032 .426$ por indemnización de daños patrimoniales y $\$ 350.000 .000$ por indemnización de daño moral, así como a la inmediata devolución de todas las garantías proporcionadas por la empresa contratista, en particular las boletas bancarias de garantía emitidas por el Banco de Crédito e Inversiones y por el Banco Security, cauciones que individualiza, con costas de la causa.

A fojas 137 el abogado don Hernán Cárdenas Delgado, en representación de "Serviu Región del Bío-Bío”, contestó la demanda, pidiendo su rechazo, con costas, basado en que no habrían tenido lugar los incumplimientos contractuales en que halla base la acción instaurada por la empresa contratista, oponiendo además la excepción perentoria de prescripción extintiva de dicha acción judicial. Adicionalmente, dedujo demanda reconvencional en contra de "Ingecol S.A., fundado en que las garantías proporcionadas por ésta no cubrirían la totalidad de las multas aplicadas administrativamente, de suerte que pide que se condene a la compañía demandada reconvencionalmente a enterar la cifra añadida de $\$ 10.000 .000$, así como a proporcionar otras boletas bancarias de garantía del buen comportamiento de las obras y a indemnizar el retardo en el cumplimiento de esta última obligación, con costas.

A fojas 158 el abogado don Fernando Saenger Gianoni, en representación de Ingecol S.A., contestó la demanda reconvencional planteada en los autos, solicitando su rechazo, con costas.

A fojas 178, después de culminado el período de discusión y de fracasada la gestión de conciliación, el tribunal de la primera instancia recibió la causa a prueba, fijando, en la calidad de sustanciales, pertinentes y controvertidos, un listado de veintiún hechos. 
A fojas 190 el tribunal de primer grado, pronunciándose acerca de recursos de reposición deducidos por las partes a fojas 180 y 183, modificó la formulación de ciertos hechos y con los numerales 22 a 24 agregó, con idénticas calidades, tres circunstancias fácticas; finalmente, esta Corte, resolviendo a fojas 574 acerca de los recursos de apelación subsidiariamente deducidos por las partes en contra de la resolución que recibió la causa a prueba, la confirmó.

Terminada la fase probatoria y después de haberse citado a las partes a oír sentencia a fojas 583 , la sentenciadora de la primera instancia dictó la sentencia definitiva de 31 de marzo de 2004, que corre a fojas 596 y siguientes, en la que rechazó la excepción de prescripción extintiva opuesta por "Serviu Región del Bío-Bío, así como las demandas deducidas por Ingecol S.A. y Serviu Región del Bío-Bío, sin costas. A fojas 596 y 606 las partes dedujeron sus recursos de apelación en contra de la recién aludida sentencia definitiva de primera instancia.

A fojas 625, con fecha 9 de junio de 2008, se dictó sentencia definitiva de primera instancia, de carácter complementario.

A fojas 627, Serviu Región del Bío-Bío interpuso el recurso de apelación en contra de la sentencia definitiva complementaria que se acaba de referir.

\section{Y TENIENDO PRESENTE:}

$1^{\circ}$ ) Que la sentencia definitiva de primera instancia, para decidir como lo hizo, se basó en que no se halla acreditada en el proceso la existencia del contrato de ejecución de obra en que encuentran su base tanto la acción instaurada por Ingecol S.A., como la excepción de prescripción extintiva planteada por Serviu Región del Bío-Bío y la acción reconvencional deducida por este servicio público; razona en el sentido que si bien las partes se hallan contestes en la existencia del contrato en cuestión, de suerte que, en principio, tal hecho debiera quedar relevado de prueba y establecido en el fallo, se trata, en la especie, de una causa en que está comprometido el interés público, de manera que el tribunal no puede proceder de ese modo sin constatar la existencia de las formalidades previstas por el ordenamiento jurídico para que tal contrato pueda tenerse por existente, echándose de menos, en el caso, tanto la constancia de haberse tramitado por la Contraloría General de la República la resolución administrativa emitida por el demandado, como la suscripción notarial por el contratista de tres transcripciones de dicha resolución y subsecuente protocolización, exigidas tales formalidades por el artículo 46 del Decreto Supremo No 355, de 1976, de Vivienda y Urbanismo.

$2^{\circ}$ ) Que no cabe duda que estos razonamientos, contenidos en la sentencia definitiva de primera instancia, se encuentran arreglados a derecho.

$3^{\circ}$ ) Que, desde el punto de vista sustantivo, debe tenerse en consideración que todas las acciones deducidas por Ingecol S.A. tienen necesaria base en la existencia de un 
contrato de ejecución de obra, consentido por las partes en los términos previstos en el artículo $1445 \mathrm{~N}^{\mathrm{2}} 2$ del Código Civil, debiendo, en la especie, para que tal contrato pueda tenerse por existente en derecho como fuente generadora de las obligaciones contractuales que las partes denuncian como incumplidas y de las acciones que Serviu Región del Bío-Bío dice extintivamente prescritas, haberse llevado a cabo las formalidades perentoriamente exigidas por el artículo 46 del Decreto Supremo $\mathrm{N}^{\circ} 355$, de 1976, de Vivienda y Urbanismo; la carencia de dichas formalidades -que el ordenamiento jurídico aplicable impone- impide concluir en la existencia jurídica del contrato en cuestión, de las obligaciones contractuales invocadas y de las acciones de responsabilidad contractual instauradas, sin que puedan, estas últimas, entenderse prescritas extintivamente, porque sólo puede extinguirse por un modo legal lo que previamente ha nacido a la vida jurídica.

$\left.4^{\circ}\right)$ Que, en perspectiva procesal, como correctamente lo apunta la consideración tercera de la sentencia definitiva de primer grado, no es posible tener por establecida la existencia del contrato de ejecución de obra en cuestión sobre la base de la ausencia de contradicción de las partes, que efectivamente se hallan contestes en su existencia, desde que la regla del artículo 313 del Código de Procedimiento Civil no es bastante a este fin, por hallarse comprometido el interés público y resultar éste predominante.

$5^{\circ}$ ) Que si bien es cierto que, en el orden procesal civil, "en cuanto a la posición de la situación de hecho, el juez, en lugar de tener que ajustarse estrictamente a la realidad, ha de acomodarse a las afirmaciones de las partes, en términos tales que "la afirmación de las partes vincula al juez en cuanto a la posición del hecho, de manera que cabe concluir que el sentenciador "no puede dejar de poner (en la sentencia) una situación de hecho que haya sido afirmada por todas las partes (Carnelutti, Francesco, La Prueba Civil, Editorial Depalma, 2000, traducción de Niceto Alcalá Zamora y Castillo de la segunda edición italiana, página 9), no lo es menos que este entendimiento constituye únicamente la regla general, puesto que se sustenta en "el poder de disposición de las partes sobre el material de hecho de la sentencia y en la admisibilidad de la influencia de las partes "sobre el contenido de la sentencia misma: las partes pueden, bien callando un hecho real o bien afirmando acordes un hecho imaginario, constreñir al juez a poner en la sentencia un hecho diverso de la realidad (Carnelutti, idem);

$6^{\circ}$ ) Que, en cambio, "en los casos en que las partes no tienen el poder de disponer sobre el estado o sobre la relación jurídica que constituye el objeto del juicio (Micheli, Gian, La Carga de la Prueba, Editorial Temis, 1989, traducción de Santiago Sentís Melendo, página 223), predomina el interés público, "de manera que el juez no está vinculado por los allegata partium (Micheli, idem);

$7^{\circ}$ ) Que, por consiguiente, la regla contenida en el artículo 313 del Código de Procedimiento Civil, en cuanto excluye de la necesidad de prueba a los hechos aceptados por las partes, se asienta sobre el implícito presupuesto de tratarse de una causa 
en que se debate acerca de relaciones jurídicas de cuyos contenidos los representantes judiciales de las partes pueden disponer libre y discrecionalmente, en los términos previstos en el artículo 12 del Código Civil; en cambio, en un caso en que el representante judicial de una de las partes carece de esa posibilidad de amplia disposición, se impone la necesidad de prueba, desde que entenderlo en sentido contrario conllevaría -en la realidad concreta- la posibilidad de eludir las reglas perentorias de derecho que impiden esa libre disponibilidad; en otros términos, la norma del artículo 313 del Código de Procedimiento Civil sólo puede aplicarse en los casos en que los representantes judiciales de las partes son titulares de facultades para transigir acerca del objeto del juicio en los términos que esas potestades son exigidas por los artículos 2447 y 2448 del Código Civil, porque, en la medida en que la vinculación del juez a la admisión fáctica común de las partes puede conducir al mismo efecto que un contrato de transacción, las mismas facultades han de ser exigidas a dicho efecto procesal.

$8^{\circ}$ ) Que, en el caso, conforme a lo dispuesto en el artículo 17, letra m), del Decreto Supremo No 355, de 1976, de Vivienda y Urbanismo, que contiene el "Reglamento Orgánico de los Servicios de Vivienda y Urbanización, el Director de Serviu Región del Bío-Bío carece de la facultad de transigir por sí mismo, desde que, para ello, requiere de autorización previa, que ha de contenerse en un decreto supremo, de manera que la norma del artículo 313 del Código de Procedimiento Civil no puede aplicarse en esta causa en el sentido de excluir de la necesidad de prueba a los hechos que -siendo sustanciales y pertinentes para la operación de las leyes decisorias de la litis- hayan sido admitidos por todas las partes.

$9^{\circ}$ ) Que, por los motivos de que se ha dejado constancia, los razonamientos sustantivos y adjetivos contenidos en la parte considerativa de la sentencia definitiva de primera instancia, se arreglan a derecho.

$10^{\circ}$ ) Que, no obstante, la referida sentencia de primera instancia adolece de vicio de casación formal.

$\left.11^{\circ}\right)$ Que, conforme al $N^{\circ} 9^{\circ}$ del artículo 768 del Código de Procedimiento Civil, es vicio de casación formal la circunstancia de "haberse faltado a algún trámite o diligencia declarados esenciales por la ley, y, con arreglo al $\mathrm{N}^{\circ} 3^{\circ}$ de su artículo 795 , es trámite o diligencia esencial en los juicios de mayor cuantía "el recibimiento de la causa a prueba cuando proceda con arreglo a la ley.

$\left.12^{\circ}\right)$ Que, como surge claramente de lo que se ha expresado en las consideraciones precedentes de este fallo, la resolución que recibió la causa a prueba debió comprender como hechos sustanciales y pertinentes, que han debido tener el mismo tratamiento que un hecho controvertido, las circunstancias de haber consentido las partes en la celebración del contrato de ejecución de obra en que se sustentan las acciones deducidas por Ingecol S.A. y Serviu Región del Bío-Bío y la excepción perentoria de prescripción 
extintiva opuesta por este último, y de haberse observado en su celebración las formalidades legalmente exigibles, así como el contenido estipulado para la contratación.

$13^{\circ}$ ) Que, pese a tal necesidad, lo cierto es que la resolución que recibió la causa a prueba a fojas 178, modificada en primer grado a fojas 190 y confirmada por esta Corte a fojas 574, no comprendió las circunstancias fácticas aludidas en el motivo precedente.

$14^{\circ}$ ) Que el vicio de casación formal previsto en las reglas legales recién aludidas se configura no sólo en el caso en que el tribunal o mite en absoluto la recepción de la causa a prueba, sino, también, en el evento en que, habiendo recibido la causa a prueba, no lo ha hecho con los contenidos que ha debido incluir para ajustarse a la ley procesal (Rioseco, Emilio, La Prueba Ante La Jurisprudencia, Editorial Jurídica de Chile, 2002, cuarta edición actualizada, página 154, N²51).

$1^{\circ}$ ) Que el vicio de casación formal cuya concurrencia se ha anotado ha cobrado en el proceso una influencia decisiva, desde que la resolución que recibió la causa a prueba bien pudo conducir a las partes a entender que se hallaban relevadas de la carga procesal de acreditar las circunstancias fácticas que se han descrito, en tanto que la sentencia definitiva de la primera instancia, ulteriormente, desechó todas sus pretensiones, precisamente sobre la base de no obrar en el proceso probanzas suficientes acerca de esos aspectos de hecho, de manera que el proceso sustanciado no ha ofrecido a los litigantes la seguridad jurídica que es necesaria para que concurran los caracteres de racionalidad y justicia que exige la garantía fundamental asegurada por el artículo $19 \mathrm{~N}^{\circ} 3$ de la Constitución Política, omisión que impone la nulidad formal de la sentencia definitiva de primera instancia de 31 de marzo de 2004, que corre a fojas 596, así como del fallo complementario que rola a fojas 625 .

16) Que el inciso $1^{\circ}$ del artículo 775 del Código de Procedimiento Civil dispone que "pueden los tribunales, conociendo por vía de apelación, consulta o casación o en alguna incidencia, invalidar de oficio las sentencias cuando los antecedentes del recurso manifiesten que ellas adolecen de vicios que dan lugar a la casación en la forma, debiendo oír sobre este punto a los abogados que concurran a alegar en la vista de la causa e indicar a los mismos los posibles vicios sobre los cuales deberán alegar.

$17^{\circ}$ ) Que esta Corte se halla conociendo de esta causa en virtud de las apelaciones interpuestas por las partes a fojas 596, 606 y 627.

$18^{\circ}$ ) Que consta del estampado que rola a fojas 674 que los abogados que concurrieron a la vista de la causa fueron llamados a alegar acerca del vicio de casación formal en cuestión y así procedieron.

$\left.1^{\circ}\right)$ Que los incisos $1^{\circ}$ y $2^{\circ}$ del artículo 786 del Código de Procedimiento Civil dispone que "en los casos de casación en la forma, la misma sentencia que declara la casación 
determinará el estado en que queda el proceso, el cual se remitirá para su conocimiento al tribunal correspondiente y que "este tribunal es aquel a quien tocaría conocer del negocio en caso de recusación del juez o jueces que pronunciaron la sentencia casada.

Por estas consideraciones y teniendo además presente lo dispuesto en las reglas legales citadas, se decide de oficio:

Que se invalida la sentencia definitiva de primera instancia de treinta y uno de marzo de dos mil cuatro, que corre a fojas 596 y siguientes, y se anula la sentencia definitiva de primera instancia, complementaria, de nueve de junio de dos mil ocho, escrita a fojas 625, además de lo obrado desde fojas 178 en adelante, reponiéndose el proceso al estado de recibírselo a prueba, debiendo dictarse por el juez no inhabilitado que corresponda la resolución que dispone el artículo 318 del Código de Procedimiento Civil, salvando la omisión en que se ha incurrido, y continuarse la sustanciación de la causa en conformidad a derecho. Sin perjuicio, se deja válidas las actuaciones y resoluciones que dicen relación con delegaciones de mandato judicial.

Regístrese y devuélvase, con su custodia.

Redacción de la Ministro Suplente doña Flora Adriana Sepúlveda Rivas.

No firma el Ministro don Jaime Solís Pino, no obstante haber concurrido a la vista de la causa y al acuerdo, por encontrarse haciendo uso de feriado legal.

\section{Comentario}

La sentencia arriba transcrita será objeto de un doble análisis: por un lado, uno técnico-jurídico acerca de la corrección de la decisión de los jueces de la instancia, y, por el otro, uno del tipo político-procesal acerca de los poderes otorgados a los jueces para administrar justicia en nuestro país y su correcto ejercicio en un Estado democrático de Derecho.

Comencemos por el primero de los análisis. Los razonamientos de la Corte (considerandos quinto a octavo) pueden ser resumidos de la siguiente manera: para que las partes puedan admitir la existencia de un hecho y dejarlo exento de actividad probatoria, es necesario que puedan disponer de los derechos e intereses objeto de la controversia. En consecuencia, cuando el proceso versa sobre derechos e intereses indisponibles -como en el presente caso- las partes carecen de toda posibilidad de admitir un hecho a la luz del Art. 313 del Código de Procedimiento Civil.

Este razonamiento presenta dos particularidades. En primer lugar, tiene el mérito de vincular la alegación de los hechos a la disponibilidad de los derechos controvertidos en pleito. Así, habría una relación entre la facultad de disposición que tiene el titular del derecho subjetivo y que se encuentra reconocida constitucionalmente, y la alegación de los elementos fácticos de una pretensión. La relación es exacta, y constituye un 
gran paso en el perfecto entendimiento del principio dispositivo como máxima que acompaña al carácter privado de los derechos subjetivos e intereses legítimos. Si en el plano extraprocesal se reconoce al ciudadano la posibilidad de disponer libremente de un derecho sustantivo, esa característica no se pierde por la existencia del proceso. Más bien, el carácter privado y disponible del derecho se robustece con la decisión del justiciable de dar inicio al proceso, de perfilar su contenido mediante la alegación de unos hechos debidamente calificados y de fijar su límite a través de la petición de una expresa tutela y de un bien de la vida. Así entonces el principio dispositivo se conecta con la esfera material del proceso, esto es, con los derechos e intereses objeto de su tutela más que con su desarrollo procedimental. El hecho de que un proceso civil tenga un objeto disponible no debería generar consecuencias en los aspectos estrictamente procesales, puesto que éstos miran al desarrollo del ejercicio jurisdiccional.

En segundo lugar, el fallo transcrito da a entender que la simple afirmación de la existencia del contrato importa disponer del derecho. O dicho en otros términos, que todo reconocimiento de un hecho constituye un acto de disposición del derecho. En el caso concreto, que el representante del órgano público reconozca la existencia del contrato es una disposición de la relación jurídica material. La afirmación no es correcta puesto que el dato fáctico que sirve de base a la pretensión no es únicamente el contrato sino que también el incumplimiento del mismo. El hecho constitutivo de la pretensión tiene un carácter complejo que se conforma por la alegación de una relación jurídica sustantiva y la afirmación precisa de la existencia de un incumplimiento a esa relación. El contrato es condición necesaria pero no suficiente del acogimiento de la pretensión. Para que esto suceda debe alegarse uno o más hechos -adicionales al contrato- que fundamenten el incumplimiento, y del cual emane el interés para accionar. Si esto es así, entonces creo que la sola aceptación del contrato no involucra disponer de la relación material sino se aceptan igualmente los hechos que justifican un incumplimiento de dicho acuerdo. En términos estrictamente procesales, sólo el reconocimiento de un hecho que constituya la lesión del derecho y justifique la necesidad de tutela jurisdiccional, es apto para hablar de disposición de la relación material. Con esto integramos un elemento netamente procesal -como el interés para accionar- dentro de idea de disposición judicial de los derechos mediante la actividad alegatoria ${ }^{1}$. Reconocer un hecho no envuelve la disposición de la relación sustantiva sino cuando ese hecho es condición necesaria y suficiente de la estimación de la pretensión. En consecuencia, aun cuando estemos en presencia de una relación patrimonial indisponible -como lo asevera la sentencia-, la aceptación de la existencia del contrato no puede ser considerada como un reconocimiento a la lesión del derecho que nace de ese contrato.

\footnotetext{
${ }^{1}$ Hay cierta paz doctrinal al momento de identificar los elementos que conforman el principio dispositivo y los actos que son muestra de su actuación en el proceso civil: la renuncia, desistimiento, allanamiento, el avenimiento, etc. Vid., Picó i Junoy, Joan: El derecho a la prueba en el proceso civil, Bosch Editor, Barcelona, 1996, p. 217 y siguientes. Esta paz no se alcanza cuando se trata de explorar las derivaciones o consecuencias netamente procesales del carácter disponible de los derechos.
} 
Quizá la muestra más palmaria de la necesidad de ir separando la naturaleza del hecho aceptado o reconocido es la amplia cabida que nuestro ordenamiento da a las convenciones probatorias, incluso en procesos que versan sobre bienes indisponibles como los de familia y penal. Para nuestro legislador el carácter indisponible de un derecho no es impedimento para que las partes puedan alcanzar acuerdos acerca de la existencia de un hecho. Sólo lo será cuando el hecho dado por cierto pueda afectar el interés público que fundamenta la indisponibilidad del derecho. Si todos los extremos de la pretensión quedaran resueltos mediante la simple aceptación de ambos litigantes entonces claramente habría una posibilidad de burlar el interés general que pueda existir en una controversia. Pero este no es el caso comentado, en que los extremos más relevantes de la pretensión, esto es, la existencia misma del incumplimiento, jamás fueron aceptados y, por el contrario, fueron el foco de la controversia. Por lo expuesto, no parece razonable que pueda excluirse la posibilidad de aceptar un hecho a través de voluntad común de darlo por verdadero por la sola circunstancia de que se trata de un asunto con objeto indisponible.

Otro tema, por cierto, es la carga de la prueba y la eficacia probatoria de ese reconocimiento. En el primer aspecto es evidente que la necesidad de tutela se satisface con la simple afirmación de que el derecho está lesionado, correspondiendo al demandado la prueba del cumplimiento íntegro y oportuno de la prestación, o de su extinción por otra causa. Pero la sola circunstancia de asumir una carga probatoria no dice absolutamente nada respecto a la disposición de la relación jurídica sustantiva. En el segundo aspecto, cuando el legislador ha dispuesto una determinada formalidad ad probationem para la eficacia judicial de un acuerdo contractual, el rechazo de todo otro medio de prueba que no consista en el cumplimiento de esa formalidad es correcto ${ }^{2}$. Para ello no hay necesidad de recurrir a la indisponibilidad del objeto del proceso. Aquí opera una exclusión probatoria por razones sustantivas.

Tal como lo anuncié, el otro aspecto que me interesa analizar de esta sentencia tiene una connotación político-procesal. El fallo de primer grado rechaza la demanda por estimar que no se había acreditado la existencia del contrato aun cuando ambas partes estaban de acuerdo en su existencia. La Corte, por su parte, acogiendo el razonamiento del juez a quo, casa de oficio en la forma la sentencia por estimar que se habría omitido un trámite y diligencia esencial como fue la agregación dentro de los puntos de prueba la "existencia de la relación contractual". Este no es un punto donde pueda haber una mayor discusión. El problema es que luego de varios años de tediosa y costosa tramitación el proceso vuelve a una etapa relativamente inicial.

\footnotetext{
${ }^{2}$ Cabe precisar que la sentencia es particularmente confusa al momento de determinar si las referidas formalidades que exige el Art. 46 del Decreto Supremo N 355, de 1976, de Vivienda y Urbanismo, son a título de solemnidad o probatorias. Creo, sin embargo, que si se hubiesen estimado como solemnidades el derrotero seguido por el fallo es bastante ilógico. Habría sido suficiente fundamentar el rechazo de la demanda en la sola circunstancia de no estar acreditada la existencia de la solemnidad.
} 
La pregunta que me surge es: ¿pudo haberse resuelto este juicio de otra forma? Entiendo que sí y es ahí donde quiero apuntar. Si los jueces de la instancia hubiesen utilizado sus poderes probatorios decretando una medida para mejor resolver entonces este pleito habría sido mucho más eficiente desde la perspectiva de la utilización del recurso público y más justo desde la realización efectiva de los derechos de los ciudadanos. De ambas cuestiones quisiera dar cuenta, colocando un especial énfasis no tanto en el procedimiento civil actual sino más bien en el proceso civil que viene.

En primer término, no cabe duda que si el Estado asegura al justiciable una respuesta jurisdiccional a sus controversias esa respuesta debe darse dentro de un tiempo razonable. La dilación injustificada de la tutela equivale a su privación, cuestión que se agrava con el procedimiento civil vigente que no cuenta con herramientas eficaces para hacer frente al factor tiempo; me refiero concretamente a la incapacidad del actual sistema cautelar para cubrir las situaciones de peligro en la demora o tardanza. El actual Proyecto de Código Procesal Civil asegura al ciudadano que la resolución de sus disputas patrimoniales se hará por medio de un juicio oral, opción que para un Estado como el nuestro que cuenta con recursos limitados es mucho más costosa que un procedimiento escrito. Por ende, es razonable exigir del proceso civil que se encamina, las herramientas necesarias para que situaciones como la sucedida en el fallo trascrito no acontezcan. Ni el ciudadano medio ni el Estado están en condiciones económicas de soportar la tramitación de procesos inútiles o innecesarios ${ }^{3}$. En el caso concreto, si el órgano jurisdiccional hubiese decretado una prueba de oficio, el dispendio económico para lograr un pronunciamiento sobre el contenido de la pretensión hubiese sido menor (más racional), tanto para el ciudadano como para el aparato jurisdiccional. Por este motivo, es necesario que nuestros jueces asuman íntegramente la naturaleza e importancia de sus poderes, y la necesidad de actuarlos para lograr los distintos fines que se persiguen a través de la jurisdicción.

El segundo aspecto digno de comentar es el real alcance de las medidas para mejor resolver como instrumento para alcanzar resultados probatorios que se acerquen en términos de correspondencia con la verdad de los hechos. Esta indagación es apenas pertinente en el procedimiento civil en vigor, puesto que existiendo un sistema tasado de valoración de la prueba los resultados probatorios están predeterminados por el legislador y poco o nada cabe hablar de verdad. Sin embargo, esto no sucede cuando se hace referencia al proceso civil que se proyecta, que otorga al juez una libertad en la valoración de prueba (sana crítica), colocándolo a la par con los procesos orales vigentes en otras ramas del Derecho (penal, familia y del trabajo).

\footnotetext{
${ }^{3}$ De ahí que el Proyecto contempla la posibilidad de un control prematuro de los presupuestos procesales e incluso la posibilidad de rechazar in limine una demanda cuando fuera manifiestamente infundada. Ambos son casos donde la completa tramitación de un proceso resulta innecesaria o inútil. Sobre este último, véase: Hunter, I.: "El poder del juez para rechazar in limine la demanda por manifiesta falta de fundamento", en Ius et Praxis, Universidad de Talca, año 15, N², 2009, pp. 117 a 163.
} 
En los procesos nacionales en que la iniciativa y recepción de la prueba son orales, las medidas para mejor resolver como modalidad propia de la actividad probatoria del tribunal, están en franca retirada. Es cierto que históricamente han gozado de una pésima fama y que nuestra jurisprudencia apartándose del texto legal que las consagra ha ido reduciendo su ámbito de aplicación ${ }^{4}$, pero eso no significa que debamos prescindir de la actividad probatoria del juez y de estas medidas en particular. Creo visualizar tres poderosas razones para entender que el nuevo proceso civil tiene que dotar al juez de poderes en materia probatoria.

En primer lugar, entiendo que la actividad probatoria del juez ayuda o contribuye -aunque no asegura- a alcanzar una decisión basada en la verdad de los hechos del litigio, entendida ésta como una verdad racional y relativa que constituye una condición mínima y necesaria para que la decisión que se adopte sea legítima y justa. Lo que se persigue con la prueba del juez es aumentar las posibilidades de que lo que se da por probado, se acerque en términos de correspondencia lo más posible a los hechos tal como sucedieron $^{5}$. La prueba dispuesta por el juez debe intentar corregir las exageraciones de la utilización del método dialéctico en la recolección del material de conocimiento, puesto que la actividad probatoria de las partes al ser eminentemente interesada (y está bien que así sea), puede mitigar las posibilidades de que el hecho que se da por probado tenga una relación de correspondencia con la realidad. En este sentido, el criterio que se debe utilizar para aportar prueba en la audiencia preliminar es el de la plenitud del material probatorio. Se trata de que todas las fuentes de prueba relevantes que han sido denunciadas por las partes, ingresen al proceso, primeramente, a instancia de parte, y luego, en forma subsidiaria o complementaria, por el juez. Es obvio, lógico y sensato sostener que los litigantes sólo se mueven por sus propios intereses, por ende, tratarán de no asumir grandes riesgos respecto a la prueba, no aportando una fuente del cual ignoren cuál será su resultado. Sólo aquellas que le ofrezcan una expectativa razonable de resultado favorable serán ingresadas al proceso. Este razonamiento, sin embargo, no es el

${ }^{4}$ Si revisamos la jurisprudencia que se ha ido articulando a partir del año 1912, donde se dicta el primer fallo sobre el Art. 159 del CPC, podemos ver cómo se ha ido restringiendo el ámbito de aplicación de las medidas para mejor resolver, reduciéndolas: a.- Finalidad de complemento o aclaración de la prueba rendida por las partes; b.- O bien, estableciendo presupuestos o condiciones para que sean decretadas, como la existencia de un antecedente serio que pueda ser esclarecido por medio de las medidas (C. Valdivia, 12 de julio de 1912, Gaceta Jurídica, T. I, No 601, p. 991; C. Santiago, 13 de junio de 1953, R.D.J., t. 49, sec. $2^{\text {a }}$, p. 144 y C. Suprema, 31 de agosto de 1970, R.D.J., t. 67, sec. 1a, p. 366). Por su parte, la doctrina nacional considera que estas diligencias han de decretarse cuando se está en presencia de un principio de prueba, lo que, en términos negativos, indicaría que el juez no puede proponer prueba cuando el hecho a probar esté huérfano de alguna actividad probatoria de las partes. Vid., Tavorali, Raúl: "Diligencias para mejor proveer: antiguos y nuevos problemas", en Tribunales, jurisdicción y proceso, Editorial Jurídica de Chile, Santiago, 1994, pp. 40 y siguientes. De más está decir que esta mala fama y práctica traspasa las fronteras de nuestro país: en España, por ejemplo, con la vigencia de la LEC 1881 las denominadas "diligencias para mejor proveer", fueron catalogadas por cierta parte de la doctrina como verdaderas "corruptelas" que sólo servían para dilatar la sentencia final.

${ }^{5}$ Vid., Taruffo, Michele: La prueba, Marcial Pons, Madrid, 2008, pp. 28 y siguientes, y Ferrer, Jordi: La valoración racional de la prueba, Marcial Pons, Madrid, 2007, pp. 29 y siguientes. 
que tiene el juez al momento de aportar prueba. Al órgano jurisdiccional le es indiferente el resultado concreto de la misma, aun cuando puede hacer un juicio fundado en la utilidad y grado de eficacia de la misma. Esta es la razón que justifica que sea el juez quien ingrese ciertas pruebas al proceso y no las partes.

En segundo lugar, hay que precisar que el enunciado probatorio, es el resultado de una sumatoria del material de conocimiento que no admite sustitución o reemplazo. Esto quiere decir que si cambian los elementos sobre los que se realiza el juicio de hecho, agregando o quitando un material de conocimiento, podría cambiar también el resultado ${ }^{6}$. Esto implica, como señala Ferrer ${ }^{7}$, que el resultado de la valoración de la prueba es siempre contextual, referido a un conjunto de elementos de juicio. Por tanto, la prueba del juez serviría para enriquecer, al menos cuantitativamente, ese conjunto de elementos de juicio sobre el que va a operar el juicio sobre los hechos.

En tercer lugar, me parece que es hora que nuestra judicatura entienda y asuma que la función que cumplen sobrepasa la mera resolución de contiendas jurídicas que supuso durante el siglo XIX la articulación de un juez pasivo. Por el contrario, la función de los tribunales no sólo está limitada a resolver un conflicto (que es el presupuesto necesario pero no suficiente del ejercicio jurisdiccional) sino a tutelar los derechos de los ciudadanos por medio de la aplicación del derecho objetivo al caso concreto, de manera de hacer efectivos los mandatos generales y abstractos contenidos en las normas jurídicas. Esta función entiende que los presupuestos fácticos de las normas jurídicas sean verdaderamente reconstruidos. El triunfo del derecho como medio de regulación de las conductas humanas depende, en gran término, de la capacidad de los tribunales de acercarse a la verdad. Sólo hay incentivo al cumplimiento espontáneo del derecho si el proceso judicial tiene la capacidad y finalidad de determinar de modo verdadero quién ha cumplido y quién no ha cumplido con el mandato legal.

Lógicamente, cualquiera sea la opción acerca del contenido e intensidad de estos poderes deberá hacerse con pleno respeto de los derechos del justiciable, por sobre todo la imparcialidad del juzgador y el derecho de defensa ${ }^{8}$.

Por último, creo que las medidas para mejor resolver podrían cumplir una utilidad diversa a la que tradicionalmente se le adjudica. La posibilidad de decretar de oficio una prueba una vez obtenidos los resultados probatorios levanta alguna sospecha acerca de la conservación de la imparcialidad subjetiva del juez que la decreta. Sin embargo,

\footnotetext{
${ }^{6}$ Que mejor ejemplo de esta afirmación que la sentencia citada, puesto que si el juicio sobre los hechos hubiese operado sobre un material de conocimiento enriquecido con la fuente de prueba que faltó ingresas entonces el resultado de la decisión hubiese sido distinta.

${ }^{7}$ Ferrer, Jordi: op. cit., p. 40.

${ }^{8}$ Cabe precisar que la potestad para aportar prueba tal como está consagrada en el Proyecto de Código Procesal Civil es dudosa en cuanto al efectivo respeto del derecho de defensa. En efecto, de acuerdo a una interpretación sistemática de este cuerpo normativo, el juez puede aportar prueba en la audiencia preliminar, momento en que la oportunidad de las partes para ese mismo objetivo ya precluyó. Con esto, se ve coartado el derecho de defensa entendido como la posibilidad de deducir contraprueba a la propuesta por el juez. Seguramente será uno de los extremos que tendrá que ser ampliamente discutido.
} 
cuando el fundamento de esa prueba es posibilitar la práctica de medios frustrados que han sido previamente propuestos a instancia de las partes o del juez, o bien renovar la práctica de otros medios cuyos resultados han sido absurdos o inconducentes, no veo inconveniente de ningún tipo para que, guardando la necesaria celeridad y concentración procedimental, pueda disponerse in extremis la práctica de la prueba. Incluso nada impide que bajo los supuestos indicados sean las partes las que propongan la renovación final de cierta actividad probatoria. 